\title{
INVARIANT SUBSPACES: CONTINUOUS STABILITY IMPLIES SMOOTH STABILITY
}

\author{
LYLE NOAKES AND KIN YAN CHUNG
}

(Communicated by Palle E. T. Jorgensen)

\begin{abstract}
The stability of an invariant subspace is defined in the terms of the existence of a certain type of function. The imposition of further conditions on this function leads to different forms of stability. Of these, the equivalence of continuous and smooth stability is proved; two proofs are offered for comparison.
\end{abstract}

\section{INTRODUCTION}

Roughly speaking, an invariant subspace $M$ of an operator $A$ on a Hilbert space is stable if invariant subspaces near $M$ exist for all operators near $A$. By requiring that the nearby subspaces meet other conditions, we can define different types of stability. Some of these forms of stability have been studied by Gohberg et al. [2] and Noakes [6].

The present work concerns proving the equivalence of two types of stability, namely, continuous and smooth stability in finite-dimensional complex Hilbert spaces. We shall provide two proofs: one using elementary linear techniques and a shorter proof using more advanced nonlinear methods.

In $\S 2$ we develop the tools we shall need. In the process, the seemingly different approaches in $[2,6]$ for defining stability are reconciled and a common basis is established for the definition of many types of stability. The abovementioned proofs are then given in $\S \S 3$ and 4 respectively.

Throughout, $H$ is assumed to be a finite-dimensional complex Hilbert space. The set of invariant (closed linear) subspaces of an operator $A$ on a Hilbert space will be denoted Lat $A$. Inner products in $H$ are denoted $\langle\cdot, \cdot\rangle$ and the (orthogonal) projection onto the subspace $M$ is denoted $P_{M}$. Given Hilbert spaces $K_{1}$ and $K_{2}$, we shall write $\mathscr{L}\left(K_{1}, K_{2}\right)$ and $\mathscr{C}\left(K_{1}\right)$ respectively for the Banach space of (bounded linear) operators $T: K_{1} \rightarrow K_{2}$ and the set of subspaces of $K_{1}$. For convenience, we write $\mathscr{L}(H)$ in place of $\mathscr{L}(H, H)$. Let $\rho$ denote the metric on $\mathscr{C}(H)$ defined by identifying subspaces with projections and referring to the operator norm; thus, $\rho(N, M)=\left\|P_{N}-P_{M}\right\|$. It is easily verified that by suitably restricting the domain of $\rho$, we obtain the corresponding metric on $\mathscr{C}(K)$ for any subspace $K$ of $H$. We always assume the operator

Received by the editors February 12, 1992.

1991 Mathematics Subject Classification. Primary 47A15. 
norm topology on $\mathscr{L}(H)$ and the metric topology on $\mathscr{C}(H)$. Finally, we shall write $\left.f\right|_{E}$ for the restriction of the function $f$ to the set $E$.

\section{Preliminaries}

From now on, $A$ is assumed to be an operator on $H$ and $M$ an invariant subspace of $A$. Stability is defined in [2] as follows: $M$ is stable if for every $\varepsilon>0$ there is a $\delta>0$ such that every operator $B$ satisfying $\|B-A\|<\delta$ has an invariant subspace $N$ with $\rho(N, M)<\varepsilon$. Equivalently, $M$ is stable if there is a function $G: V \rightarrow \mathscr{C}(H)$ defined on a neighbourhood $V$ of $A$ such that $G$ is continuous at $A, G(A)=M$, and $G(B) \in$ Lat $M$ for all $B \in V$. On the other hand, the definition in [6] requires the existence of a $C^{1}$ function $F: V \rightarrow \mathscr{L}\left(M, M^{\perp}\right)$ defined near $A$ satisfying $F(A)=0$ and $(1+F(B)) M \in$ Lat $B$ for all $B \in V$; here, $(1+T) M=\{x+T x: x \in M\}$.

Let us make the

Definition 1. An invariance function for $(A, M)$ is a function $F: V \rightarrow$ $\mathscr{L}\left(M, M^{\perp}\right)$ such that $F(A)=0$ and $(1+F(B)) M \in$ Lat $B$ for all $B \in V$, where $V$ is a neighbourhood of $A$. We say that $M$ is a stable $A$-invariant subspace if there exists an invariance function $F$ for $(A, M)$ that is continuous at $A$. Moreover, we say that $M$ is smoothly stable (respectively continuously stable) if $F$ can be chosen to be $C^{1}$ (respectively continuous).

Implicit to the following result is a homeomorphism between $\{N \in \mathscr{C}(H)$ : $\rho(N, M)<1\}$ and $\mathscr{L}\left(M, M^{\perp}\right)$ which allows us to conclude that $M$ is stable if and only if it is stable in the sense of [2].

Proposition 1 (see [1, Theorem 1]). Let $M$ and $N$ be subspaces of $H$. The following are equivalent.

(1) $\left\|P_{N}-P_{M}\right\|<1$.

(2) $N=(1+T) M$ for some $T \in \mathscr{L}\left(M, M^{\perp}\right)$.

If any of the above conditions holds, then $T$ is unique and satisfies

$$
\|T\|=\frac{\left\|P_{N}-P_{M}\right\|}{\sqrt{1-\left\|P_{N}-P_{M}\right\|^{2}}} .
$$

Indeed, if we refer to the structure of a $C^{\infty}$-manifold for $\mathscr{C}(H)$ given in [1], we may use the alternative

Definiton 2. A function $G: V \rightarrow \mathscr{C}(H)$ defined on a neighbourhood of $A$ is called an invariance function for $(A, M)$ if $G(A)=M$ and $G(B) \in$ Lat $B$ for all $B \in V$. We say that $M$ is stable if there is an invariance function for $(A, M)$ that is continuous at $A$. Moreover, $M$ is continuously stable (respectively smoothly stable) if the invariance function can be chosen to be continuous (respectively $C^{1}$ ).

The invariance function of Definitions 1 and 2 are related by homeomorphism, and we shall use the two definitions interchangeably; the applicable definition can be inferred from the codomain specified.

Smooth stability is clearly stronger than continuous stability, so it suffices to prove

Theorem 1. Continuous stability implies smooth stability.

We shall need the following results in the latter sections. 
Lemma 1. Let $M$ be a nontrivial subspace of $H$, and let $A \in \mathscr{L}(H)$. Suppose that, relative to the decomposition $H=M \oplus M^{\perp}, A$ has the matrix representation

$$
A=\left[\begin{array}{ll}
A_{11} & A_{12} \\
A_{21} & A_{22}
\end{array}\right],
$$

where $A_{11} \in \mathscr{L}(M), A_{12} \in \mathscr{L}\left(M^{\perp}, M\right), A_{21} \in \mathscr{L}\left(M, M^{\perp}\right)$, and $A_{22} \in$ $\mathscr{L}\left(M^{\perp}, M^{\perp}\right)$. If $T \in \mathscr{L}\left(M, M^{\perp}\right)$, then $(1+T) M \in$ Lat $A$ if and only if $A_{21}+A_{22} T=T A_{11}+T A_{12} T$.

Proof. Relative to the decomposition $H=M \oplus M^{\perp},(1+T) M$ is the graph of $T$. Now, the image of $(x, T x)$ under $A$ is $\left(A_{11} x+A_{12} T x, A_{21} x+A_{22} T x\right)$, which is in $(1+T) M$ if and only if $A_{21} x+A_{22} T x=T\left(A_{11} x+A_{12} T x\right)$. Therefore, $(1+T) M \in$ Lat $A$ if and only if $\left(A_{21}+A_{22} T\right) x=T\left(A_{11}+A_{12} T\right) x$ for all $x \in M$.

Before giving the next result, we need a definition. Recall that, if $X$ is a topological space and $\left\{E_{n}\right\}_{n=1}^{\infty}$ is a sequence of subsets of $X$, then $\lim _{n \rightarrow \infty} \inf _{n \rightarrow \infty} E_{n}$ is the set of all limits of sequences $\left\{x_{n}\right\}_{n=1}^{\infty}$ with $x_{n} \in E_{n}$ and $\lim \sup _{n \rightarrow \infty} E_{n}$ is the set of all limits of subsequences of sequences $\left\{x_{n}\right\}_{n=1}^{\infty}$ with $x_{n} \in E_{n}$.

Definition 3. We say that a sequence $\left\{M_{n}\right\}_{n=1}^{\infty}$ of subspaces of $H$ converges semistrong to a subspace $M$ if

$$
\liminf _{n \rightarrow \infty} M_{n}=\limsup _{n \rightarrow \infty} M_{n}=M \text {. }
$$

Proposition 2. Metric convergence, strong convergence, and semistrong convergence in $\mathscr{C}(H)$ are equivalent.

Proof. A result of Halmos [4, Theorem 1] shows that strong convergence and semistrong convergence are equivalent. Since $H$ is finite dimensional, metric convergence is the same as strong convergence.

From now on, $\Xi$ denotes the operator on $\mathscr{L}\left(M, M^{\perp}\right)$ defined by

$$
\Xi(T)=T A_{11}-A_{22} T
$$

for all $T \in \mathscr{L}\left(M, M^{\perp}\right)$.

Theorem 2. Let $H$ be a complex finite-dimensional Hilbert space, and let $M$ be an invariant subspace of an operator $A$ in $\mathscr{L}(H)$. Then the following are equivalent.

(1) $M$ is smoothly stable.

(2) $\Xi$ is surjective.

(3) $\sigma\left(A_{11}\right) \cap \sigma\left(A_{22}\right)=\varnothing$.

Proof. (1) implies (2). This is a special case of [6, Theorem 1].

(2) implies (3). We shall prove the contrapositive. Suppose $\sigma\left(A_{11}\right) \cap \sigma\left(A_{22}\right) \neq$ $\varnothing$, and let $\lambda \in \sigma\left(A_{11}\right) \cap \sigma\left(A_{22}\right)$. Then $A_{11}-\lambda$ is not injective and $A_{22}-\lambda$ is not surjective. Choose $u \in \operatorname{ker}\left(A_{11}-\lambda\right) \backslash\{0\}$ and $v \in M^{\perp} \backslash \operatorname{ran}\left(A_{22}-\lambda\right)$, and define $T \in \mathscr{L}\left(M, M^{\perp}\right)$ by $T x=\langle x, u\rangle v$. For all $S \in \mathscr{L}\left(M, M^{\perp}\right)$ we have $\Xi(S)=S A_{11}-A_{22} S=S\left(A_{11}-\lambda\right)-\left(A_{22}-\lambda\right) S$, so $\Xi(S) u=-\left(A_{2}-\lambda\right) S u \in$ $\operatorname{ran}\left(A_{22}-\lambda\right)$. Therefore, $\Xi(S) u \neq T u$; whence, $\Xi$ is not surjective.

(3) implies (1). This is a special case of [6, Theorem 2]. 
If $H$ is a complex Hilbert space, then the spectrum $\sigma(A)$ of an operator $A$ on $H$ is the set of eigenvalues of $A$. Given $\lambda \in \sigma(A)$, we shall denote the root subspace of $A$ corresponding to the eigenvalue $\lambda$ by $R_{\lambda}(A)$, thus,

$$
R_{\lambda}(A)=\bigcup_{k=1}^{\infty} \operatorname{ker}(A-\lambda)^{k}
$$

A standard result tells us that

$$
M=\bigoplus_{\lambda \in \sigma(A)}\left(M \cap R_{\lambda}(A)\right)
$$

for all $A$-invariant subspaces $M$.

Proposition 3 (see [2, Theorem 15.1.4]). Let $M \in$ Lat $A$, and let $E$ be an open subset of $C$ such that $\sigma\left(\left.A\right|_{M}\right) \subseteq E$. If $B \in \mathscr{L}(H), N \in$ Lat $B$, and $\rho(N, M)+\|B-A\|$ is sufficiently small, then $\sigma\left(\left.B\right|_{N}\right) \subseteq E$.

Proposition 4 (see [2, Theorem 15.2.1]). Let $\lambda_{1}, \ldots, \lambda_{p}$ be the different eigenvalues of a transformation $A$ in $\mathscr{L}(H)$. A subspace $M$ of $H$ is $A$-invariant and stable if and only if $M=\bigoplus_{j=1}^{p} M_{j}$ where each $M_{j}$ is an arbitrary $A$-invariant subspace of $R_{\lambda_{j}}(A)$ if $\operatorname{dim} \operatorname{ker}\left(A-\lambda_{j}\right)=1$; if $\operatorname{dim} \operatorname{ker}\left(A-\lambda_{j}\right) \neq 1$ then either $M_{j}=\{0\}$ or $M_{j}=R_{\lambda_{j}}(A)$.

Proposition 5 (see [2, p. 449]). A subspace $M$ is a stable invariant subspace of $A$ if and only if it is an isolated $A$-invariant subspace. Equivalently, $M$ is stable if and only if there exists an $r>0$ such that $(1+T) M \notin$ Lat $A$ for all nonzero $T \in \mathscr{L}\left(M, M^{\perp}\right)$ satisfying $\|T\|<r$.

\section{AN ELEMENTARY PROOF USING LINEAR METHODS}

Our first approach to proving Theorem 1 is to first reduce the problem to the case where the operator has only one eigenvalue. The following two lemmas achieve this goal.

Lemma 2. Suppose $H$ is a direct sum $\bigoplus_{j=1}^{p} K_{j}$ of subspaces, and let $M$ be a subspace of $H$ satisfying $M=\bigoplus_{j=1}^{p}\left(M \cap K_{j}\right)$. Given $\varepsilon>0$, there exists $a$ $\delta>0$ such that if $N$ is a subspace of $H$ for which $N=\bigoplus_{j=1}^{p}\left(N \cap K_{j}\right)$ and if $\rho(N, M)<\delta$ then $\rho\left(N \cap K_{j}, M \cap K_{j}\right)<\varepsilon$ for $j=1, \ldots, p$.

Proof. Suppose the assertion is false, so that there is an $\varepsilon>0$ and a sequence $\left\{M_{n}\right\}_{n=1}^{\infty}$ of subspaces such that $M_{n}=\bigoplus_{j=1}^{p}\left(M_{n} \cap K_{j}\right), M_{n} \rightarrow M$, and $\rho\left(M_{n} \cap K_{q}, M \cap K_{q}\right) \geq \varepsilon$ for some $q$ (possibly depending on $n$ ). By extracting an appropriate subsequence if necessary, we can assume without loss of generality that $q$ is fixed and that $\rho\left(M_{n} \cap K_{q}, M \cap K_{q}\right) \geq \varepsilon$ for all $n$.

Now $M=\liminf _{n \rightarrow \infty} M_{n}$ by Proposition 2, so if $x \in M \cap K_{q}$, then there is a sequence $\left\{x_{n}\right\}_{n=1}^{\infty}$ with $x_{n} \in M_{n}$ such that $x_{n} \rightarrow x$. Each $x_{n}=\sum_{j=1}^{p} x_{n j}$, where $x_{n j} \in M_{n} \cap K_{q}$, so applying the projection onto $K_{q}$ along the direct sum of the remaining $K_{j}$ yields $x_{n q} \rightarrow x$; whence, $x \in \liminf _{n \rightarrow \infty} M_{n} \cap K_{q}$. Therefore,

$$
M \cap K_{q} \subseteq \liminf _{n \rightarrow \infty} M_{n} \cap K_{q} .
$$


Conversely, $\lim \sup _{n \rightarrow \infty} M_{n} \cap K_{q} \subseteq \lim \sup _{n \rightarrow \infty} M_{n}=M$ and $\lim \sup _{n \rightarrow \infty} M_{n} \cap$ $K_{q} \subseteq \lim \sup _{n \rightarrow \infty} K_{q}=K_{q}$; whence,

$$
\limsup _{n \rightarrow \infty} M_{n} \cap K_{q} \subseteq M \cap K_{q}
$$

Consequently $\rho\left(M_{n} \cap K_{q}, M \cap K_{q}\right) \rightarrow 0$ by Proposition 2, contradicting $\rho\left(M_{n} \cap K_{q}, M \cap K_{q}\right) \geq \varepsilon$.

Lemma 3. Suppose $M$ is a continuously stable A-invariant subspace, and let $\lambda \in \sigma(A)$. Then $M \cap R_{\lambda}(A)$ is a continuously stable $\left.A\right|_{R_{\lambda}(A)}$-invariant subspace.

Proof. Let $K_{1}=R_{\lambda}(A)$, and let $K_{2}$ be the direct sum of the root subspaces of $A$ other than $R_{\lambda}(A)$. Then $A=\left.\left.A\right|_{K_{1}} \oplus A\right|_{K_{2}}$ relative to the direct sum $H=K_{1} \oplus K_{2}$. The eigenvalues of $A$ are isolated, so there is an open subset $E$ of $C$ such that $\sigma(A) \cap E=\{\lambda\}$. Since $\sigma\left(\left.A\right|_{K_{1}}\right)=\{\lambda\} \subseteq E$, there is a neighbourhood $V_{0}$ of $\left.A\right|_{K_{1}}$ in $\mathscr{L}\left(K_{1}\right)$ such that $\sigma(B) \subseteq E$ for all $B \in V_{0}$. Note that $\sigma(B) \cap \sigma\left(\left.A\right|_{K_{2}}\right)=\varnothing$ and $K_{1}=\bigoplus_{\mu \in \sigma(B)} R_{\mu}(B)$ for all $B \in V_{0}$.

Let $F^{\prime}: V^{\prime} \rightarrow \mathscr{C}(H)$ be a continuous invariance function for $(A, M)$, where $V^{\prime}$ is a neighbourhood of $A$. Put $W=V^{\prime} \cap\left\{\left.B \oplus A\right|_{K_{2}}: B \in V_{0}\right\}$. Then $\left.F^{\prime}\right|_{W}: W \rightarrow \mathscr{C}(H)$ is continuous. Since $K_{1}$ is a spectral subspace of every $B^{\prime} \in W$, it follows that $F^{\prime}\left(B^{\prime}\right) \cap K_{1} \in$ Lat $\left.B^{\prime}\right|_{K_{1}}$ for all $B^{\prime} \in W$. Put $V=\{B \in$ $\left.V_{0}:\left.B \oplus A\right|_{K_{2}} \in V^{\prime}\right\}$, and define $F: V \rightarrow \mathscr{C}\left(R_{\lambda}(A)\right)$ by $F(B)=F^{\prime}\left(\left.B \oplus A\right|_{K_{2}}\right) \cap K_{1}$. Then $F(B) \in$ Lat $B$ for all $B \in V$.

If $B \in V$, then any $\left(\left.B \oplus A\right|_{K_{2}}\right)$-invariant subspace $N$ satisfies $N=(N \cap$ $\left.K_{1}\right) \oplus\left(N \cap K_{2}\right)$. Fix $B_{0} \in V$; then $\left\|\left(\left.B \oplus A\right|_{K_{2}}\right)-\left(\left.B_{0} \oplus A\right|_{K_{2}}\right)\right\|=\left\|B-B_{0}\right\|$. Let $N=F^{\prime}\left(\left.B \oplus A\right|_{K_{2}}\right)$ and $N_{0}=F^{\prime}\left(\left.B_{0} \oplus A\right|_{K_{2}}\right)$. Given $\varepsilon>0$, Lemma 2 asserts the existence of $\varepsilon^{\prime}>0$ such that $\rho\left(N, N_{0}\right)<\varepsilon^{\prime}$ implies $\rho\left(N \cap K_{1}, N_{0} \cap K_{1}\right)<\varepsilon$. But there is a $\delta>0$ such that $\left\|B-B_{0}\right\|<\delta$ implies $\rho\left(N, N_{0}\right)<\varepsilon^{\prime}$; whence, $\rho\left(F(B), F\left(B_{0}\right)\right)=\rho\left(N \cap K_{1}, N_{0} \cap K_{1}\right)<\varepsilon$, so $F$ is continuous on $V$. Observe that $F\left(\left.A\right|_{R_{\lambda}(A)}\right)=M \cap R_{\lambda}(A)$, so $M \cap R_{\lambda}(A)$ is continuously stable.

ihe following result allows us to keep track of eigenvalues systematically.

Lemma 4. Suppose $M$ is a continuously stable invariant subspace of $A$, and let $F: V \rightarrow \mathscr{C}(H)$ be a continuous invariance function for $(A, M)$. Let $\Gamma:[0,1] \rightarrow$ $V$ be continuous, and let $\lambda_{1}, \ldots, \lambda_{n}$ be continuous complex-valued functions such that the eigenvalues of $\Gamma(\alpha)$ are $\lambda_{1}(\alpha), \ldots, \lambda_{n}(\alpha)$ with (algebraic) multiplicities counted, for all $\alpha \in[0,1]$. For each $\alpha \in[0,1]$, put

$$
\Omega_{\alpha}=\left\{j \in\{1, \ldots, n\}: \lambda_{j}(\alpha) \in \sigma\left(\left.\Gamma(\alpha)\right|_{F(\Gamma(\alpha))}\right)\right\} .
$$

If $\left|\Omega_{\alpha}\right|$ is constant for all $\alpha \in[0,1]$, then the $\Omega_{\alpha}$ are all equal.

Proof. In what follows, $\alpha$ and $\beta$ are always understood to be elements of $[0,1]$, and $j$ is always assumed to be in $\{1, \ldots, n\}$. Given $\alpha$, choose disjoint open sets $E_{\alpha}$ and $E_{\alpha}^{\prime}$ such that

$$
\sigma\left(\left.\Gamma(\alpha)\right|_{F(\Gamma(\alpha))}\right) \subseteq E_{\alpha} \text { and } \sigma(\Gamma(\alpha)) \backslash \sigma\left(\left.\Gamma(\alpha)\right|_{F(\Gamma(\alpha))}\right) \subseteq E_{\alpha}^{\prime} .
$$

Thus $\sigma(\Gamma(\alpha)) \subseteq E_{\alpha} \cup E_{\alpha}^{\prime}$, and $\lambda_{j}(\alpha) \in E_{\alpha}$ if and only if $j \in \Omega_{\alpha}$, for all $j$.

Now, there exists an $r_{\alpha}>0$ such that if $|\beta-\alpha|<r_{\alpha}$, then $\sigma(\Gamma(\beta)) \subseteq E_{\alpha} \cup E_{\alpha}^{\prime}$. Because $E_{\alpha}$ and $E_{\alpha}^{\prime}$ are disjoint and since $\left\{\lambda_{j}(\beta): \beta \in\left(\alpha-r_{\alpha}, \alpha+r_{\alpha}\right) \cap[0,1]\right\}$ is connected for each $j$, it follows that $\lambda_{j}(\beta) \in E_{\alpha}$ if and only if $j \in \Omega_{\alpha}$, for all $j$ and for all $\beta \in\left(\alpha-r_{\alpha}, \alpha+r_{\alpha}\right) \cap[0,1]$. 
The map $\beta \mapsto \rho(F(\Gamma(\beta)), F(\Gamma(\alpha)))+\|\Gamma(\beta)-\Gamma(\alpha)\|$ is continuous, so by Proposition 3 , there exists an $s_{\alpha}>0$ such that $|\beta-\alpha|<s_{\alpha}$ implies $\sigma\left(\left.\Gamma(\beta)\right|_{F(\Gamma(\beta))}\right) \subseteq E_{\alpha}$.

Put $\delta_{\alpha}=\min \left\{r_{\alpha}, s_{\alpha}\right\}$, and suppose $|\beta-\alpha|<\delta_{\alpha}$. If $j \in \Omega_{\beta}$, then $\lambda_{j}(\beta) \in$ $\sigma\left(\left.\Gamma(\beta)\right|_{F(\Gamma(\beta))}\right) \subseteq E_{\alpha}$, so $j \in \Omega_{\alpha}$; hence, $\Omega_{\beta} \subseteq \Omega_{\alpha}$. Since $\left|\Omega_{\beta}\right|=\left|\Omega_{\alpha}\right|$, the two sets are equal.

Letting $\alpha$ vary, we obtain an open cover $\left\{\left(\alpha-\delta_{\alpha}, \alpha+\delta_{\alpha}\right) \cap[0,1]: \alpha \in[0,1]\right\}$ for $[0,1]$. By Lebesgue's Covering Lemma, there exists an $\varepsilon>0$ such that, for every $\beta$, there is an $\alpha$ satisfying $[\beta, \beta+\varepsilon] \cap[0,1] \subseteq\left(\alpha-\delta_{\alpha}, \alpha+\delta_{\alpha}\right) \cap[0,1]$. In particular, if we choose $p$ so large that $1 / p<\varepsilon$, then there exist $\alpha_{0}, \ldots, \alpha_{p-1}$ such that

$$
[q / p,(q+1) / p] \subseteq\left(\alpha-\delta_{\alpha_{q}}, \alpha+\delta_{\alpha_{q}}\right)
$$

for $q=0, \ldots, p-1$. Consequently, $\Omega_{q / p}=\Omega_{\alpha_{q}}=\Omega_{(q+1) / p}$; successively, letting $q=0, \ldots, p-1$, we see that $\Omega_{(q+1) / p}=\Omega_{0}$ for each $q$. Finally, every $\beta$ is in some $[q / p,(q+1) / p]$, so $\Omega_{\beta}=\Omega_{\alpha_{q}}=\Omega_{(q+1) / p}=\Omega_{0}$. We conclude that the $\Omega_{\alpha}$ are all equal.

If the eigenvalues $\lambda_{1}(\alpha), \ldots, \lambda_{n}(\alpha)$ are distinct for each $\alpha$ and $\operatorname{dim} F(\Gamma(\alpha))$ is constant, then $\left|\Omega_{\alpha}\right|$ is also constant since each subscript $j$ contributes $\operatorname{dim}\left(F(\Gamma(\alpha)) \cap R_{\lambda_{j}(\alpha)}(\Gamma(\alpha))\right)$ elements to $\Omega_{\alpha}$.

Lemma 5. Let $A \in \mathscr{L}(H)$, and suppose $\sigma(A)=\{\lambda\}$. Then the only continuously stable A-invariant subspaces are trivial.

Proof. If $\operatorname{dim} H=1$, there is nothing to prove, so we assume $\operatorname{dim} H>1$. Suppose $M$ is a continuously stable $A$-invariant subspace. Then $M$ is obviously stable. By hypothesis $\sigma(A)=\{\lambda\}$, so $R_{\lambda}(A)=H$ and, by Proposition 4, $M$ is trivial if $\operatorname{dim} \operatorname{ker}(A-\lambda) \neq 1$.

It remains to consider the case where $\operatorname{dim} \operatorname{ker}(A-\lambda)=1$. Here, the Jordan decomposition of $A$ consists of exactly one Jordan block, so there is a basis $\left\{v_{1}, \ldots, v_{n}\right\}$ for $H$ relative to which $A$ has the matrix

$$
\left[\begin{array}{ccccc}
\lambda & 1 & \cdots & 0 & 0 \\
0 & \lambda & \cdots & 0 & 0 \\
\vdots & \vdots & \ddots & \vdots & \vdots \\
0 & 0 & \cdots & \lambda & 1 \\
0 & 0 & \cdots & 0 & \lambda
\end{array}\right] .
$$

Let $F: V \rightarrow \mathscr{C}(H)$ be a continuous invariance function for $(A, M)$. Define the operator $Z \in \mathscr{L}(H)$ by

$$
Z v_{j}= \begin{cases}v_{n} & \text { if } j=1, \\ 0 & \text { otherwise. }\end{cases}
$$

Let $\mu \in \mathbf{C}$; then $A+\mu Z$ has the matrix

$$
\left[\begin{array}{ccccc}
\lambda & 1 & \cdots & 0 & 0 \\
0 & \lambda & \cdots & 0 & 0 \\
\vdots & \vdots & \ddots & \vdots & \vdots \\
0 & 0 & \cdots & \lambda & 1 \\
\mu & 0 & \cdots & 0 & \lambda
\end{array}\right]
$$

relative to the basis $\left\{v_{1}, \ldots, v_{n}\right\}$. 
Put $\mu=\nu^{n}$; then straightforward calculation shows that $A+\mu Z$ has $n$ distinct eigenvalues, namely, $\lambda+\omega^{j} \nu, j=1, \ldots, n$, with corresponding eigenvectors having coordinates $\left(1, \omega^{j} \nu, \ldots,\left(\omega^{j} \nu\right)^{n-1}\right)$. Here, $\omega=e^{i 2 \pi / n}$ is a primitive $n$th root of unity. Without loss of generality we may assume that $\lambda=0$ (since $\operatorname{Lat}(B-\lambda)=\operatorname{Lat} B$ for all $B \in \mathscr{L}(H)$ ), so the eigenvalues of $A+\mu Z$ are $\omega^{j} \nu, j=1, \ldots, n$.

We can choose $r>0$ small enough so that $A+\mu Z \in V$ and $\operatorname{dim} F(A+\mu Z)=$ $\operatorname{dim} M$, if $|\mu|=r^{n}$. Define $\Gamma:[0,1] \rightarrow V$ by $\Gamma(\alpha)=A+r^{n} e^{i 2 \pi \alpha} Z$, and let $\lambda_{1}, \ldots, \lambda_{n}$ be the complex-valued functions defined on $[0,1]$ by $\lambda_{j}(\alpha)=$ $r \omega^{j} e^{i 2 \pi \alpha / n}$. Then $\lambda_{1}(\alpha), \ldots, \lambda_{n}(\alpha)$ are the eigenvalues of $\Gamma(\alpha)$. Let $\Omega_{\alpha}$ be defined as in Lemma 4. Then the $\Omega_{\alpha}$ are equal $\left(\left|\Omega_{\alpha}\right| \equiv \operatorname{dim} M\right.$ since the eigenvalues of $\Gamma(\alpha)$ have multiplicity one each).

It is obvious that $\Gamma(0)=\Gamma(1)$. If $j \in \Omega_{0}=\Omega_{1}$, then $r \omega^{j}=\lambda_{j}(0) \in$ $\sigma\left(\left.\Gamma(0)\right|_{F(\Gamma(0))}\right)$ and $r \omega^{j+1}=\lambda_{j}(1) \in \sigma\left(\left.\Gamma(1)\right|_{F(\Gamma(1))}\right)=\sigma\left(\left.\Gamma(0)\right|_{F(\Gamma(0))}\right)$; whence, $(j+1 \bmod n) \in \Omega_{0}$. Consequently, either $\Omega_{0}=\varnothing$ or $\Omega_{0}=\{1, \ldots, n\}$; that is, either $\operatorname{dim} M=0$ or $\operatorname{dim} M=n$. Hence $M$ is trivial.

We are now ready to prove Theorem 1 .

Proof of Theorem 1. Suppose to the contrary that $M$ is a continuously stable but not smoothly stable $A$-invariant subspace. Then $\sigma\left(A_{11}\right) \cap \sigma\left(A_{22}\right) \neq \varnothing$ by Theorem 2. Let $\lambda \in \sigma\left(A_{11}\right) \cap \sigma\left(A_{22}\right)$. Then $M \cap R_{\lambda}(A)$ is a nontrivial subspace of $R_{\lambda}(A)$. On the other hand, $M \cap R_{\lambda}(A)$ is a continuously stable $\left.A\right|_{R_{\lambda}(A)^{-}}$ invariant subspace by Lemma 3; whence, $M \cap R_{\lambda}(A)=\{0\}$ or $M \cap R_{\lambda}(A)=$ $R_{\lambda}(A)$ by Lemma 5 . This is a contradiction.

\section{A PROOF USING NONLINEAR METHODS}

We now present an alternative proof of Theorem 1 which involves the calculation of the topological degree of various maps.

Alternative proof of Theorem 1 . Suppose $M$ is continuously stable, and let $F: V_{0} \rightarrow \mathscr{L}\left(M, M^{\perp}\right)$ be a continuous invariance function for $(A, M)$. Suppose $A$ has the matrix representation given in Lemma 1 . Note that $A_{21}=0$ because $M \in$ Lat $A$. Given $S \in \mathscr{L}\left(M, M^{\perp}\right)$, let $B(S)$ be the operator on $H$ with matrix representation

$$
\left[\begin{array}{cc}
A_{11} & A_{12} \\
S & A_{22}
\end{array}\right]
$$

relative to the decomposition $H=M \oplus M^{\perp}$. By Lemma $1,(1+T) M \in \operatorname{Lat} B(S)$ if and only if

$$
T A_{12} T+T A_{11}-A_{22} T-S=0,
$$

or equivalently

$$
T A_{12} T+T A_{11}-A_{22} T=S .
$$

By Proposition 5, we can choose $r>0$ such that $(1+T) M \notin$ Lat $A$ for all $T \in \mathscr{L}\left(M, M^{\perp}\right)$ with $0<\|T\|<r$. In terms of Lemma 1, this means that

$$
T A_{12} T+T A_{11}-A_{22} T \neq 0 \text {. }
$$

Put $V=\left\{T \in V_{0}:\|T\|<r\right\}$, and let $W=\left\{S \in \mathscr{L}\left(M, M^{\perp}\right): B(S) \in V\right\}$. Define $g: W \backslash\{0\} \rightarrow \mathscr{L}\left(M, M^{\perp}\right) \backslash\{0\}$ by $g(S)=F(B(S))$. If $S \neq 0$, then clearly $M \notin$ Lat $B(S)$, so $g(S) \neq 0$ and $g$ is well defined. 
Let $f: V \backslash\{0\} \rightarrow \mathscr{L}\left(M, M^{\perp}\right) \backslash\{0\}$ be defined by $f(T)=T A_{12} T+T A_{11}-$ $A_{22} T$. Our choice of $r$ ensures that $f$ is well defined. Moreover, $f(g(S))=S$ by equation (1) since $(1+g(S)) M \in$ Lat $B(S)$.

Since $f$ and $g$ are continuous functions mapping punctured neighbourhoods of 0 in $\mathscr{L}\left(M, M^{\perp}\right)$ into punctured neighbourhoods of 0 in $\mathscr{L}\left(M, M^{\perp}\right)$, they have well-defined topological degrees $\operatorname{deg} f$ and $\operatorname{deg} g$. We have seen that $f \circ g$ is the identity map, so $\operatorname{deg} f \times \operatorname{deg} g=\operatorname{deg} f \circ g=1$.

Now assume to the contrary that $M$ is not smoothly stable. The linear terms of $f(T)$ are given by $\Xi(T)$, and by Theorem $2, \Xi$ is not surjective and hence is singular. If we choose a basis for $\mathscr{L}\left(M, M^{\perp}\right)$, then $f$ reduces to $p$ polynomials $\pi_{1}, \ldots, \pi_{p}$ in $p$ complex variables $z_{1}, \ldots, z_{p}$, where $p=m n$, $n=\operatorname{dim} H$, and $m=\operatorname{dim} M$. We can choose a linear change of coordinates so that the coefficients of $z_{1}$ in $\pi_{1}, \ldots, \pi_{p}$ are identically zero.

Invoking two results in [3, p. 670] yields $\operatorname{deg} f=\operatorname{dim} \mathscr{O} / I$, where $\mathscr{O}$ is the local ring at the origin and $I$ is the ideal generated by the $\pi_{j}$. Define the homomorphism $\phi: \mathscr{O} / I \rightarrow \mathbf{C}$ by $\phi(\theta+I)=\theta(0)$. Then $\operatorname{ker} \phi=M / I$ where $M$ is the maximal ideal of $\mathscr{O}$. However, it is clear that $z_{1}+I \in M$, so $M \neq I$ and $\operatorname{dim} \operatorname{ker} \phi \geq 1$. Consequently

$$
\operatorname{deg} f=\operatorname{dim} \mathscr{O} / I \geq 2,
$$

and then $\operatorname{deg} f \times \operatorname{deg} g=1$ cannot hold-a contradiction.

\section{REFERENCES}

1. K. Y. Chung, Subspaces and graphs, Proc. Amer. Math. Soc. 119 (1993), 141-146.

2. I. Gohberg, P. Lancaster, and L. Rodman, Invariant subspaces of matrices with applications, Wiley-Interscience, New York, 1986.

3. P. Griffiths and J. Harris, Principles of algebraic geometry, Wiley-Interscience, New York, 1978.

4. P. R. Halmos, Limsups of lats, Indiana Univ. Math. J. 29 (1980), 293-311.

5. L. Nirenberg, Topics in nonlinear functional analysis, Courant Institute of Mathematical Sciences, New York University, 1974.

6. L. Noakes, Invariant subspaces and perturbations, Proc. Amer. Math. Soc. 114 (1992), $365-370$.

Department of Mathematics, The University of Western Australia, Nedlands 6009, Australia

E-mail address, L. Noakes: lyle@maths.uwa.oz.au

Department of Mathematics, Princeton University, Princeton, New Jersey 08544

E-mail address, K. Y. Chung: kinyan@math.princeton.edu 